\title{
Non-axisymmetric Magnetorotational Instabilities in Cylindrical Taylor-Couette Flow
}

\author{
Rainer Hollerbach ${ }^{1}$, Vijaya Teeluck ${ }^{1}$ and Günther Rüdiger ${ }^{2}$ \\ ${ }^{1}$ Department of Applied Mathematics, University of Leeds, Leeds, LS2 9JT, United Kingdom \\ ${ }^{2}$ Astrophysikalisches Institut Potsdam, An der Sternwarte 16, D-14482 Potsdam, Germany
}

(Dated: May 31, 2018)

\begin{abstract}
We study the stability of cylindrical Taylor-Couette flow in the presence of azimuthal magnetic fields, and show that one obtains non-axisymmetric magnetorotational instabilities, having azimuthal wavenumber $m=1$. For $\Omega_{o} / \Omega_{i}$ only slightly greater than the Rayleigh value $\left(r_{i} / r_{o}\right)^{2}$, the critical Reynolds and Hartmann numbers are $\operatorname{Re}_{c} \sim 10^{3}$ and $\mathrm{Ha}_{c} \sim 10^{2}$, independent of the magnetic Prandtl number Pm. These values are sufficiently small that it should be possible to obtain these instabilities in the PROMISE experimental facility.
\end{abstract}

PACS numbers: 47.20.-k, 47.65.+a, 95.30.Qd

The magnetorotational instability (MRI) was discovered in 1959 by Velikhov [1], who considered cylindrical Taylor-Couette flow in the presence of an axial magnetic field, and obtained instabilities in otherwise hydrodynamically stable flows. Several decades later, it was recognized that much the same instability plays a crucial role in the dynamics of astrophysical accretion disks [2]. This prompted renewed interest in the MRI in TaylorCouette flow, and specifically in the possibility of achieving it experimentally $[3,[4,[5]$. By applying combined axial and azimuthal magnetic fields, the PROMISE facility [6, 7, 8, 9] succeeded in obtaining axisymmetric MRI modes. We show here that if the magnetic field is taken to be predominantly azimuthal, one obtains nonaxisymmetric modes that should be achievable in the PROMISE facility.

Taylor-Couette flow, the flow between differentially rotating cylinders, is one of the most fundamental problems in classical fluid dynamics [10]. A key result is the socalled Rayleigh criterion, stating that the flow will be hydrodynamically stable if the angular momentum $\Omega r^{2}$ increases outward, which occurs whenever the inner and outer cylinder's rotation rates are adjusted such that $\Omega_{o} / \Omega_{i}>\left(r_{i} / r_{o}\right)^{2}$. However, as Velikhov [1] was the first to show, such flows may nevertheless be magnetohydrodynamically unstable, provided only that the angular velocity $\Omega$ decreases outward, that is, $\Omega_{o} / \Omega_{i}<1$. This new instability, now called the magnetorotational instability, has no analog in the purely hydrodynamic problem, and arises because of the angular momentum transferred via the magnetic tension in the field lines.

As Balbus and Hawley [2] first realized, the MRI may be critically important in accretion disks, whose Keplerian angular velocity profiles, $\Omega \sim r^{-3 / 2}$, are in precisely this regime where the angular momentum increases outward but the angular velocity decreases. That is, purely hydrodynamically accretion disks would be stable [1], but magnetohydrodynamically they may be unstable, thereby accounting for the turbulence and resulting angular momentum transport that is needed to actually accrete material inward. See for example [12] for a recent review of the MRI in astrophysics.

The recognition of its astrophysical significance led to a resurgence of interest in the MRI in its original TaylorCouette context as well, in particular the possibility of studying it in laboratory experiments. Following Velikhov, it was originally suggested $[3,4]$ to impose an axial magnetic field. However, this 'standard' MRI (SMRI) has one very considerable disadvantage, namely that the rotation rates required to achieve it are enormous.

The relevant parameter turns out to be not the hydrodynamic Reynolds number $\operatorname{Re}=\Omega_{i} r_{i}^{2} / \nu$, but rather the magnetic Reynolds number $\mathrm{Rm}=\Omega_{i} r_{i}^{2} / \eta$, where $\nu$ is the viscosity and $\eta$ the magnetic diffusivity. The SMRI sets in when $\mathrm{Rm} \sim 10$. Re is then given by $\mathrm{Rm} / \mathrm{Pm}$, where $\mathrm{Pm}=\nu / \eta$ is the magnetic Prandtl number, a material property of the fluid. Typical values are $\sim 10^{-5}$ for liquid sodium, and $\sim 10^{-6}$ for gallium. Re must therefore exceed $10^{6}$ or even $10^{7}$, which unfortunately leads to increasingly strong end-effects [13]. These can perhaps be overcome [14], but the SMRI has not been obtained yet.

An alternative approach was suggested by [15], who showed that in a combined axial and azimuthal magnetic field, the relevant parameter is Re rather than $\mathrm{Rm}$ - that is, the scaling with $\mathrm{Pm}$ is altered - and that the resulting 'helical' MRI (HMRI) occurs when Re $\sim 10^{3}$, several orders of magnitude less than what would be required for the SMRI. This new design was quickly implemented in the PROMISE facility [6, 7, 8, [9], and does indeed yield modes in good agreement with the theoretical predictions. Note though that end-effects inevitably play an important role in this set-up as well, particularly due to the traveling wave nature of the HMRI. The implications for the PROMISE results continue to be debated [16, 17].

In this work we start with a purely azimuthal field. Velikhov [1] had already considered this as well, and showed that it does not yield any axisymmetric instabilities like the SMRI or the (continuously connected) HMRI. It can, however, yield non-axisymmetric instabilities, as [18] first demonstrated in an astrophysical context (where the magnetic fields in accretion disks may indeed be predominantly azimuthal rather than axial). The 
possibility of obtaining non-axisymmetric instabilities is also particularly exciting, as it would help to circumvent Cowling's theorem, disallowing purely axisymmetric dynamo action.

In the Taylor-Couette problem considered here, this 'azimuthal' MRI (AMRI) was briefly noted by [19], but only in a parameter regime that is not experimentally accessible. We show here that for rotation ratios $\Omega_{o} / \Omega_{i}$ only slightly greater than the Rayleigh limit $\left(r_{i} / r_{o}\right)^{2}$, the relevant parameters are sufficiently small that it should be achievable in the PROMISE facility.

Given the basic state consisting of an azimuthal magnetic field $\mathbf{B}_{0}=B_{0}\left(r_{i} / r\right) \hat{\mathbf{e}}_{\phi}$, imposed by running a current down the central axis, as well as an angular velocity profile $\Omega(r)$, imposed by differentially rotating the inner and outer cylinders, we begin by linearizing the governing equations about it. The perturbation flow $\mathbf{u}$ and field b may be expressed as

$$
\begin{aligned}
& \mathbf{u}=\nabla \times\left(e \hat{\mathbf{e}}_{r}\right)+\nabla \times \nabla \times\left(f \hat{\mathbf{e}}_{r}\right), \\
& \mathbf{b}=\nabla \times\left(g \hat{\mathbf{e}}_{r}\right)+\nabla \times \nabla \times\left(h \hat{\mathbf{e}}_{r}\right) .
\end{aligned}
$$

Taking the $(\phi, z, t)$ dependence to be $\exp (i m \phi+i k z+\gamma t)$, the perturbation equations become

$$
\begin{aligned}
& \operatorname{Re} \gamma\left(C_{2} e+C_{3} f\right)+C_{4} e+C_{5} f \\
& =\operatorname{Re} E_{1}+\operatorname{Re} F_{1}+\mathrm{Ha}^{2} G_{1}+\mathrm{Ha}^{2} H_{1}, \\
& \operatorname{Re} \gamma\left(C_{3} e+C_{4} f\right)+C_{5} e+C_{6} f \\
& =\operatorname{Re} E_{2}+\operatorname{Re} F_{2}+\mathrm{Ha}^{2} G_{2}+\mathrm{Ha}^{2} H_{2}, \\
& \operatorname{Rm} \gamma\left(C_{1} g+C_{2} h\right)+C_{3} g+C_{4} h \\
& \quad=E_{3}+F_{3}+\operatorname{Rm} G_{3}+\operatorname{Rm} H_{3}, \\
& \operatorname{Rm} \gamma\left(C_{2} g+C_{3} h\right)+C_{4} g+C_{5} h \\
& \quad=E_{4}+F_{4}+\operatorname{Rm} G_{4}+\operatorname{Rm}_{4} .
\end{aligned}
$$

The operators $C_{n}$ are defined by $C_{n} p=\hat{\mathbf{e}}_{r} \cdot(\nabla \times)^{n}\left(p \hat{\mathbf{e}}_{r}\right)$, and work out to be

$$
\begin{gathered}
C_{1}=0, \quad C_{2}=\Delta, \quad C_{3}=-2 m k r^{-2}, \\
C_{4}=-\Delta \partial_{r}^{2}+\left(m^{2} r^{-2}-k^{2}\right)\left(r^{-1} \partial_{r}-r^{-2}\right)+\Delta^{2}, \\
C_{5}=4 m k\left(r^{-2} \partial_{r}^{2}-r^{-3} \partial_{r}+\left(1-m^{2}\right) r^{-4}-k^{2} r^{-2}\right), \\
C_{6}=\Delta \partial_{r}^{4}-2\left(m^{2} r^{-2}-k^{2}\right) r^{-1} \partial_{r}^{3} \\
+\left(5 m^{2} r^{-4}-3 k^{2} r^{-2}-2 \Delta^{2}\right) \partial_{r}^{2} \\
+\left(3 m^{2}\left(2 m^{2}-3\right) r^{-4}+\left(4 m^{2}+3\right) k^{2} r^{-2}-2 k^{4}\right) r^{-1} \partial_{r} \\
+m^{2}\left(9-10 m^{2}\right) r^{-6}-3 k^{2} r^{-4}+2 k^{4} r^{-2}+\Delta^{3},
\end{gathered}
$$

where $\partial_{r}=\partial / \partial r$, and $\Delta=m^{2} r^{-2}+k^{2}$. The other quantities are

$$
E_{1}=-i m \Delta \Omega e, \quad E_{2}=i k \hat{\Delta} \Omega e,
$$

$$
\begin{gathered}
E_{3}=0, \quad E_{4}=i m r^{-2} \Delta e, \\
F_{1}=i k\left(\hat{\Delta} \Omega+\Delta r \Omega^{\prime}\right) f \\
F_{2}=-i m \Omega\left(C_{4}+4 k^{2} r^{-2}\right) f-i m \Delta\left(\Omega^{\prime \prime}+3 r^{-1} \Omega^{\prime}\right) f, \\
F_{3}=i m r^{-2} \Delta f, \quad F_{4}=-i k r^{-2} \hat{\Delta} f, \\
G_{1}=i m r^{-2} \Delta g, \quad G_{2}=-i k r^{-2} \hat{\Delta} g, \\
G_{3}=0, \quad G_{4}=-i m \Delta \Omega g, \\
H_{1}=-2 i m^{2} k r^{-4} h, \quad H_{2}=i m r^{-2} C_{4} h+4 i m k^{2} r^{-4} h, \\
H_{3}=-i m \Delta \Omega h, \quad H_{4}=i k\left(2 m^{2} r^{-2} \Omega-\Delta r \Omega^{\prime}\right) h,
\end{gathered}
$$

where primes denote $d / d r$, and $\hat{\Delta}=4 m^{2} r^{-2}+2 k^{2}$. All of these terms are easily derivable using MAPLE, or some other symbolic algebra package.

Length has been scaled by $r_{i}$, time by $\Omega_{i}^{-1}, \Omega$ by $\Omega_{i}, \mathbf{u}$ by $\Omega_{i} r_{i}, \mathbf{B}_{0}$ by $B_{0}$, and $\mathbf{b}$ by $\operatorname{Rm} B_{0}$. The two Reynolds numbers Re and Rm are as above; the Hartmann number $\mathrm{Ha}=B_{0} r_{i} / \sqrt{\rho \mu \eta \nu}$, where $\rho$ is the fluid's density and $\mu$ the magnetic permeability. Another parameter that appears implicitly is the rotation ratio $\hat{\mu}=\Omega_{o} / \Omega_{i}$, which enters into the details of $\Omega(r)=c_{1}+c_{2} / r^{2}$. The radius ratio is fixed at $r_{i} / r_{o}=1 / 2$, as in the PROMISE experiment.

The radial structure of $e, f, g$ and $h$ was expanded in terms of Chebyshev polynomials, typically up to $N=$ $30-60$. These equations and associated boundary conditions (no slip for $\mathbf{u}$, insulating for $\mathbf{b}$ ) then reduce to a large $(4 N \times 4 N)$ matrix eigenvalue problem, with the eigenvalue being the growth or decay rate $\gamma$ of the given mode. This numerical implementation is very different from that of [19], in which the individual components of $\mathbf{u}$ and $\mathbf{b}$ were used, and discretized in $r$ by finite differencing. Both codes yielded identical results though in every instance where we benchmarked one against the other.

Figure 1 shows the results for $m=1$, the most unstable wavenumber. At each point in the Ha-Re-plane, we repeatedly solve the basic eigenvalue problem to find the axial wavenumber $k$ that yields the largest $\operatorname{Re}(\gamma)$. We see that if $\hat{\mu}$ is only slightly greater than the Rayleigh limit 0.25 , values as small as $\mathrm{Ha} \sim 10^{2}$ and $\mathrm{Re} \sim 10^{3}$ are already sufficient to achieve instability. As $\hat{\mu}$ is increased, increasingly large values are required. Note also that these results are independent of the Prandtl number; $\mathrm{Pm}=10^{-5}, 10^{-6}$, or indeed even 0 all yield identical results (where we recall that Pm enters the equations via $\mathrm{Rm}=\mathrm{PmRe}$.

The crucial question then is whether Ha $\sim 10^{2}$ and Re $\sim 10^{3}$ are achievable in the PROMISE facility. Re $\sim$ $10^{3}$ is certainly possible; this is precisely the range where the HMRI has already been obtained. $\mathrm{Ha}=10^{2}$ is somewhat more challenging, corresponding to a current of 13 $\mathrm{kA}$ along the central axis, roughly twice what was required for the HMRI. Once the latest upgrade is complete though, currents up to $20 \mathrm{kA}$ will be achievable (F. Stefani, private communication). 

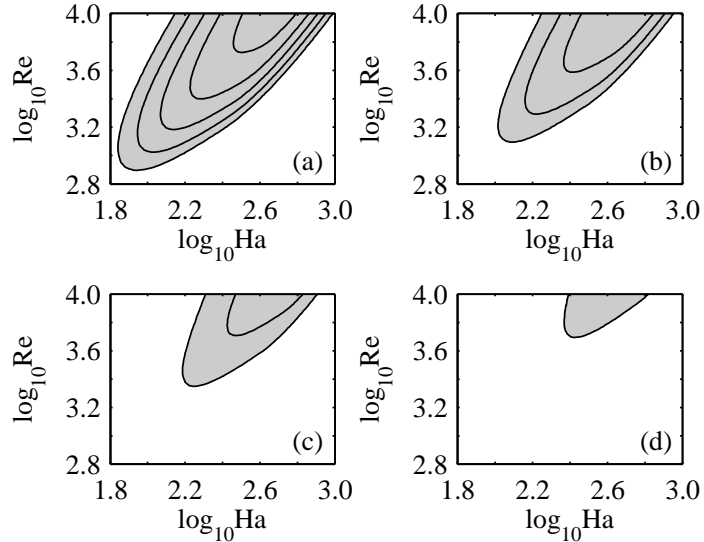

FIG. 1: The grey-shaded regions show where $\operatorname{Re}(\gamma)>0$. The contour interval is 0.01 , indicating that these instabilities grow on the basic rotational timescale $\Omega_{i}^{-1}$, but with a somewhat smaller multiplicative factor than for the SMRI. (a) $\hat{\mu}=0.25$, (b) $\hat{\mu}=0.26$, (c) $\hat{\mu}=0.27$, (d) $\hat{\mu}=0.28$.

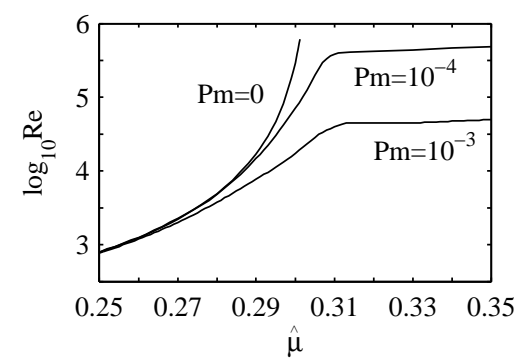

FIG. 2: $\operatorname{Re}_{c}$ as a function of $\hat{\mu}$, optimized over $k$ and Ha.

Figure 2 quantifies how the critical Reynolds number increases with $\hat{\mu}$. That is, we now optimize over Ha as well as $k$, and compute the minimum value of Re that still allows instability. The behavior is remarkably similar to the transition from the HMRI to the SMRI, as shown in Fig. 1 of [15]. In both cases $\operatorname{Re}_{c}$ is $\sim 10^{3}$, and independent of $\mathrm{Pm}$ for $\hat{\mu}$ only slightly greater than the Rayleigh value, but then increases dramatically, and scales as $\mathrm{Pm}^{-1}$ once $\hat{\mu}$ is sufficiently large.

Having obtained this non-axisymmetric instability in a purely azimuthal field, and demonstrated that it should be achievable in the PROMISE experiment, it is of further interest to add an axial field again, and investigate at what point one switches back to the previous axisymmetric HMRI. We therefore modify the equations to impose a field of the form $\mathbf{B}_{0}=B_{0}\left[\left(r_{i} / r\right) \hat{\mathbf{e}}_{\phi}+\delta \hat{\mathbf{e}}_{z}\right]$, and explore what happens as $\delta$ is increased from 0 .

At this point we must also consider the handedness of both the basic state and the resulting instabilities. For a purely azimuthal field, the basic state has no handedness, that is, it is invariant to reversing the sign of $z$. As a result, instabilities that spiral either to the left (for which $m k>0$ ) or to the right (for which $m k<0$ ),
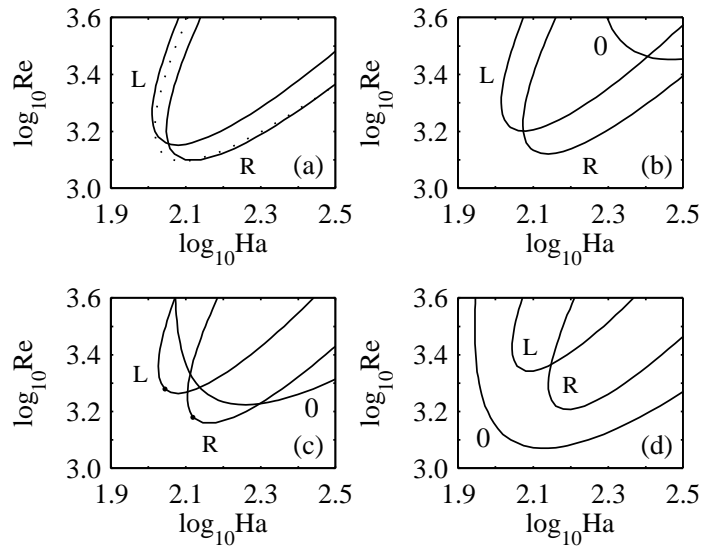

FIG. 3: The instability curves in the Ha-Re-plane, as in Fig. 1, but at fixed $\hat{\mu}=0.26$. (a) $\delta=0.02$, (b) $\delta=0.03$, (c) $\delta=0.04$, (d) $\delta=0.05$. Curves labeled L/R denote left/right spiraling $m=1$ modes, curves labeled 0 the HMRI. The dotted curve in (a) is at $\delta=0$, where the $\mathrm{L} / \mathrm{R}$ curves are identical. The two dots in (c) correspond to the solutions shown in Fig. 4.

necessarily have exactly the same critical Reynolds and Hartmann numbers. For a combined azimuthal and axial field though, the basic state itself has a handedness [15, 20], so left and right spiraling instabilities must be considered separately.

Figure 3 shows the results for $\hat{\mu}=0.26$ and $\mathrm{Pm}=0$. An axial field as weak as $\delta=0.02$ is already enough to induce a clear asymmetry between the left and right spirals, but both are otherwise still similar to the $\delta=0$ results from Fig. 1(b), included here as the dotted line. For $\delta=0.03$ another new feature emerges, the curve labeled 0 . This is precisely the previous $m=0$, axisymmetric HMRI. At this value of $\delta$ the non-axisymmetric modes are still preferred though. Further increasing $\delta$, the asymmetry between left and right spirals gradually becomes greater, and both curves shift upward slightly, indicating that these modes are suppressed by the addition of an axial field. In contrast, the HMRI is strongly excited, so much so that by $\delta=0.05$ it is already the preferred mode.

We can at least begin to understand why $m=0$ and 1 behave so differently by noting that if $m=0$ and $\delta=0$, the instability equation for the field component $h$ reduces to just free decay, $\operatorname{Rm} \gamma C_{2} h+C_{4} h=0$. However, in the absence of this part of the field $\nabla \times \nabla \times\left(h \hat{\mathbf{e}}_{r}\right)$, there is no radial component to provide the coupling between different radii that ultimately drives the MRI, since the other part of the field $\nabla \times\left(g \hat{\mathbf{e}}_{r}\right)$ has no radial component.

This is essentially Velikhov's original proof that a purely azimuthal field does not yield any axisymmetric instabilities. See also [21], who extend Velikhov's analysis from ideal to diffusive fluids. To obtain an axisymmetric instability, we therefore require $\delta \neq 0$. This couples $h$ to the other components again, thereby allowing the HMRI 

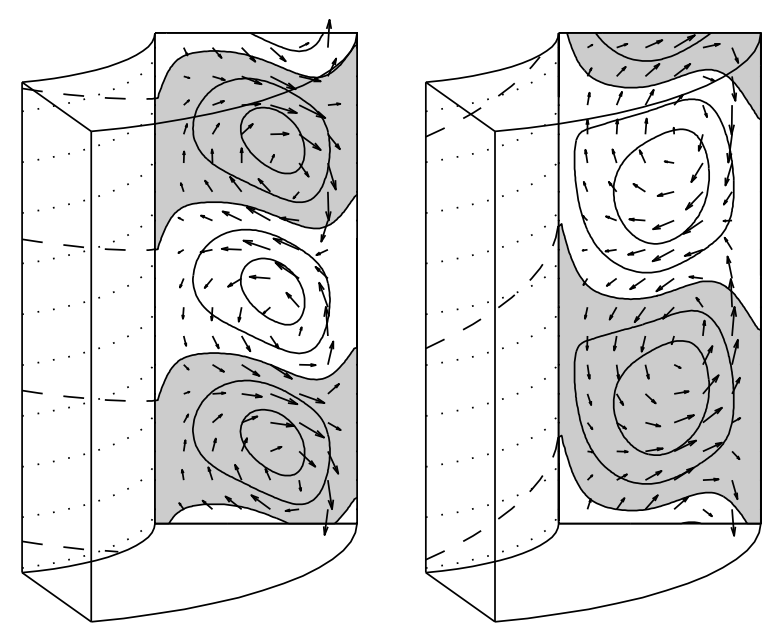

FIG. 4: The left and right spiral modes, at the two dots indicated in Fig. 3(c). On the left $\mathrm{Re}=1910$, $\mathrm{Ha}=110$, $k=4.2, \operatorname{Im}(\gamma)=-0.24$; on the right $\mathrm{Re}=1510, \mathrm{Ha}=130$, $k=-3.0, \operatorname{Im}(\gamma)=-0.27$. Arrows denote the meridional flow $\left(u_{r}, u_{z}\right)$, normalized such that the maximum $\left(u_{r}^{2}+u_{z}^{2}\right)^{1 / 2}$ is 1. Contours show $u_{\phi}$, with a contour interval of 0.2 , grey positive and white negative.

to proceed.

The key difference between $m=0$ and 1 then is that for $m=1, h$ is coupled to the other components even if $\delta=0$. A quick glance at the instability equations reveals numerous factors of $m$, and hence terms that drop out for $m=0$ but not for $m \neq 0$. It is this additional coupling that allows the non-axisymmetric AMRI to exist even in a purely azimuthal field.

Finally, Fig. 4 shows the spatial structures of the left and right spirals for $\delta=0.04$. The arrows indicate the meridional flow $\left(u_{r}, u_{z}\right)$, and the contours the azimuthal velocity $u_{\phi}$. The dashed lines on the inner cylinders denote lines of constant phase $m \phi+k z$, so depending on the sign of $k$ modes spiral either to the left or to the right. (Alternatively, one could fix $k$ to be positive, and then consider $m= \pm 1$.) The dotted lines on the inner cylinders represent a field line of the imposed, slightly helical field $\mathbf{B}_{0}$. The physical significance of the left/right asymmetry therefore is that the two modes spiral in the opposite/same direction as the imposed field.

Future work will consider the nonlinear interactions among these different modes. Exactly symmetric left and right spirals in non-magnetic Taylor-Couette flow already allow a rich variety of possibilities, including both traveling and standing waves [10, 22, 23]. It remains to be seen which of these occurs here for $\delta=0$. By judiciously adjusting $\delta$, Ha and Re, it should also be possible in this problem to preferentially select either the left or right modes, or indeed the axisymmetric HMRI. The regime $\delta \sim 0.04$, where all three modes have comparable criti- cal Hartmann and Reynolds numbers, is likely to yield particularly rich dynamics. Taylor-Couette flows in predominantly azimuthal magnetic fields of this type clearly deserve further attention, both experimental and theoretical.

This work was supported by the Science and Technology Facilities Council under Grant No. PP/E001092/1.

[1] E. P. Velikhov, Sov. Phys. JETP 36, 995 (1959).

[2] S. A. Balbus and J. F. Hawley, Astrophys. J. 376, 214 (1991).

[3] G. Rüdiger and Y. Zhang, Astron. Astrophys. 378, 302 (2001).

[4] H. T. Ji, J. Goodman, and A. Kageyama, Mon. Not. R. Astron. Soc. 325, L1 (2001)

[5] MHD Couette Flows: Experiments and Models, edited by R. Rosner, G. Rüdiger, and A. Bonanno, AIP Conf. Proc. No. 733 (AIP, New York, 2004).

[6] F. Stefani, T. Gundrum, G. Gerbeth, G. Rüdiger, M. Schultz, J. Szklarski, and R. Hollerbach, Phys. Rev. Lett. 97, 184502 (2006).

[7] F. Stefani, T. Gundrum, G. Gerbeth, G. Rüdiger, J. Szklarski, and R. Hollerbach, New J. Phys. 9, 295 (2007).

[8] F. Stefani, G. Gerbeth, T. Gundrum, J. Szklarski, G. Rüdiger, and R. Hollerbach, Astron. Nachr. 329, 652 (2008).

[9] F. Stefani, G. Gerbeth, T. Gundrum, R. Hollerbach, J. Priede, G. Rüdiger, and J. Szklarski, Phys. Rev. E 80, 066303 (2009).

[10] P. Chossat and G. Iooss, The Couette-Taylor Problem (Springer, Berlin, 1994).

[11] H. T. Ji, M. Burin, E. Schartman, and J. Goodman, Nature 444, 343 (2006).

[12] S. A. Balbus, Annu. Rev. Astron. Astrophys. 41, 555 (2003).

[13] R. Hollerbach and A. Fournier, In [5], p. 114. See also astro-ph/0506081

[14] E. Schartman, H. T. Ji, and M. J. Burin, Rev. Sci. Inst. 80, 024501 (2009).

[15] R. Hollerbach and G. Rüdiger, Phys. Rev. Lett. 95, 124501 (2005).

[16] W. Liu, Astrophys. J. 692, 998 (2009).

[17] J. Priede and G. Gerbeth, Phys. Rev. E 79, 046310 (2009).

[18] G. I. Ogilvie and J. E. Pringle, Mon. Not. R. Astron. Soc. 279, 152 (1996).

[19] G. Rüdiger, R. Hollerbach, M. Schultz, and D. Elstner, Mon. Not. R. Astron. Soc. 377, 1481 (2007).

[20] E. Knobloch, Phys. Fluids 8, 1446 (1996).

[21] I. Herron and F. Soliman, Appl. Math. Lett. 19, 1113 (2006).

[22] J. Langenberg, G. Pfister and J. Abshagen, Phys. Rev. E 68, 056308 (2003).

[23] A. Pinter, M. Lücke, and C. Hoffmann, Phys. Rev. Lett. 96, 044506 (2006). 